\title{
Improved Light Curves of LMC Eclipsing Binaries
}

\author{
Young Woon Kang, Kyungsoo Hong \\ Department of Astronomy, Sejong University, Seoul, 143-747, Korea
}

Woo-Baik Lee, Ho-Il Kim

Korea Astronomy Observatory, Taejon, 305-348, Korea

Kyu-Dong Oh

Department of Earth Sciences, Chonnam National University, Kwangju, 500-757, Korea

We present accurate BV light curves and continuum energy curves of the EROS eclipsing binaries in the Large Magellanic Cloud to find accurate binary parameters as well as their distances. The observations have been carried out using the 2.1 meter telescope in CASLEO Argentina during Feb. 1-10, 2003. We have concentrated CCD direct observations upon EROS field 1 and 2 to improve the accuracy of light curves of eclipsing binaries in the fields. The spectroscopic observations have been also carried out using the simple dispersion method to get continuum energy curves between wavelengths of $4000-8000 \AA$ for several EROS eclipsing binaries. At first, we determined a combined temperature of both components of each binary system using the continuum. Then, the combined temperature was resolved using the first estimation of the light curve solution. Finally, we determined the photometric solutions of several binaries in the Large Magellanic Cloud. 